\title{
Thyrotoxic Periodic Paralysis: A Case Report and Literature Review
}

\author{
M. J. Barahona; I. Vinagre; L. Sojo; J. M. Cubero; and Antonio Pérez
}

\begin{abstract}
We describe a 37-year-old man with a 4-month history of episodic muscular weakness, involving mainly lower-limbs. Hypokalemia was documented in one episode and managed with intravenous potassium chloride. Hyperthyroidism was diagnosed 4 months after onset of attacks because of mild symptoms. The patient was subsequently diagnosed as having thyrotoxic periodic paralysis associated with Graves' disease. Treatment with propranolol and methimazol was initiated and one year later he remains euthyroid and symptom free. Thyrotoxic periodic paralysis is a rare disorder, especially among Caucasians, but it should always be considered in patients with acute paralysis and hypokalemia, and thyroid function should be evaluated.
\end{abstract}

Keywords: Hyperthyroidism; Hypokalemia; Thyrotoxic periodic paralysis

\footnotetext{
Reprint Requests:

Antonio Pérez

Department of Endocrinology

Hospital Sant Pau

S. Antoni M. Claret 167

08025 Barcelona

Spain

Tel: +3493556566

Fax: +34935565727

E-mail: aperez@santpau.cat

Received: August 25, 2008

Revised: January 6, 2009

Accepted: January 30, 2009

doi: $10.3121 / \mathrm{cmr} .2009 .816$
}

$\mathrm{T}$ hyrotoxic periodic paralysis (TPP) is a rare complication of hyperthyroidism characterized by episodes of muscle weakness and hypokalemia. TPP is often not recognized at first attack due to a very low prevalence among the Caucasian population and usually mild symptoms of hyperthyroidism. We report a case of TPP due to Graves' disease in a Caucasian male, who presented with 4 paralytic episodes before the diagnosis was made.

\section{Case Report}

A 37-year-old Caucasian man was admitted at the hospital for evaluation of episodic muscular weakness. He recalled four similar episodes in the previous 4 months, which started during sleep or post-exercise rest. Attacks consisted of flaccid muscle weakness that varied from mild proximal leg weakness to quadriparesis, involving mainly the lower-limbs. Three attacks resolved spontaneously over 2 to 8 hours. Hypokalemia $(2.3 \mathrm{mEq} / \mathrm{L})$ and mild hypomagnesemia $(1.53 \mathrm{mg} / \mathrm{dL})$ were documented in one episode and managed with administration of intravenous potassium chloride in the emergency department. In this attack, proximal flaccid quadriparesis $2 / 5$ was observed, with normal reflexes and sensory examination. The electrocardiogram was normal.

Two weeks later (between episodes) laboratory studies revealed normal renal and hepatic function, and the following results: serum sodium $143 \mathrm{mEq} / \mathrm{L}$ (136-145), potassium $3.6 \mathrm{mEq} / \mathrm{L}$ (3.50-5.10), calcium $9.03 \mathrm{mg} / \mathrm{dL}$ (8.27-9.80), albumin $34.5 \mathrm{~g} / \mathrm{L}$ (34-48), urine sodium $77 \mathrm{mEq} / \mathrm{L}$ (25-150), urine potassium $93 \mathrm{mEq} / \mathrm{L}(17-83)$. He had no significant personal or familial medical history and he was not taking any medication. He related weight loss, distal tremor, and heat 
Table 1. Main laboratory results on admission and during follow-up.

\begin{tabular}{|c|c|c|c|c|c|c|c|}
\hline Time since diagnosis & Day 0 & Month 2 & Month 3 & Month 4 & Month 8 & Month 11 & Month 14 \\
\hline Thyrotropine $(\mu \mathrm{U} / \mathrm{mL})$ & $<0.03$ & 2.16 & 25.8 & 4.43 & 3.43 & 3.23 & 0.82 \\
\hline Free thyroxine (ng/dL) & 3.14 & 0.44 & 0.19 & 0.85 & 0.99 & 1.13 & \\
\hline Free tri-iodothyronine (ng/dL) & 1.44 & & & & & & \\
\hline Methimazole dose $(\mathrm{mg} / \mathrm{d})$ & 0 & 60 & 20 & 5 & 5 & 5 & 0 \\
\hline Serum potassium (mEq/L) & 4.38 & 4.52 & 4.19 & 4.7 & 4.38 & 4.6 & \\
\hline
\end{tabular}

Day 0 , on admission.

Normal reference levels: thyrotropine, $0.25-5.0 \mu \mathrm{U} / \mathrm{mL}$; free thyroxine, $0.77-1.71 \mathrm{ng} / \mathrm{dL}$; free tri-iodothyronine, $0.23-0.39 \mathrm{ng} / \mathrm{dL}$.

intolerance for 3 to 4 months. On admission, blood pressure was $130 / 70 \mathrm{mmHg}$ and heart-rate 75 beats/minute. Physical examination revealed a slightly enlarged thyroid gland without nodules, fine resting tremor of the hands, and mild proximal lower limb weakness. No exophtalmus or skin changes were present. Thyroid stimulating hormone level was $<0.03 \mu \mathrm{U} /$ $\mathrm{mL}$ (0.25-5.0), free thyroxin level $3.14 \mathrm{ng} / \mathrm{dL}$ (0.77-1.71), and free tri-iodothyronine $1.44 \mathrm{ng} / \mathrm{dL}(0.23-0.39)$. Thyroid radioiodine uptake was $48 \%$ at 2 hours and $72 \%$ at 24 hours, and scan revealed a diffuse homogeneous uptake. The patient was diagnosed as having thyrotoxic periodic hypokalemic paralysis associated with Graves' thyrotoxicosis. Treatment with propranolol and methimazole was initiated (table 1). After 1 year of follow-up the patient remains euthyroid and symptom free.

\section{Discussion}

The present case describes a Caucasian patient suffering from TPP due to Graves' disease. TPP is an uncommon and potentially life-threatening complication of thyrotoxicosis characterized by acute and reversible episodes of muscle weakness and hypokalemia. It occurs in about 0.1 to $0.2 \%$ of the hyperthyroid population in North America, and it is 10 times more frequent in the Oriental population and in males. ${ }^{1}$ The age of onset is usually in the third decade of life. ${ }^{2}$ The high incidence of this disorder in Asians and the association with the presence of HLA-DRw8 suggests that the basic defect may be genetically determined, but the precise pathogenesis of TPP remains unclear. ${ }^{3}$ Although the association with the HLA system may suggest an immunogenetic etiology of the TPP, some patients have thyrotoxicosis without an autoimmune mechanism, such as Jod-Basedow phenomenon, thyroiditis, thyroid stimulating hormone-secreting pituitary tumor, abuse of thyroid hormone, solitary toxic thyroid adenoma, and amiodarone-induced thyrotoxicosis. ${ }^{4}$ Although episodes of paralysis are not correlated with the severity of the thyrotoxic state, it is known that resolution of TPP occurs once euthyroidism is restored ${ }^{1-3}$ therefore, the presence of excessive thyroid hormone in serum seems necessary for this disorder. It has been shown that patients with TPP have a significantly higher Na-K-ATPase pump number and activity than healthy subjects or thyrotoxic patients without a history of paralysis. ${ }^{3}$ Thyroid hormone, $\beta$-adrenergic catecholamine and insulin can increase the pump activity in skeletal muscles, liver or kidneys. ${ }^{5}$ This leads to a shift of potassium into the cells, manifested as low serum potassium, as seen in our patient, but without changing the total body potassium level. This may explain why weakness resolves when potassium returns to the extracellular space. Hypophosphatemia and hypomagnesemia have also been reported and may contribute to the muscle weakness. $^{6}$ Finally, the attacks of paralysis tend to occur during the night, as in our patient, and after stress, alcohol intake or a carbohydrate-rich meal, suggesting a possible role of hyperinsulinemia. ${ }^{3,7}$ It has also been described that therapy with glucocorticoid at high-dose ${ }^{8}$ antiretroviral for AIDS, ${ }^{9}$ or interferon-alpha are possible precipitating factors. ${ }^{10}$

A myopathic pattern during attacks of paralysis that disappeared during remission has been reported in electromyogram, while peripheral nerve function remains normal. There are also some case-reports which describe rhabdomyolysis associated with a severe attack of TPP. ${ }^{11}$ Vacuolation and mitochondrial abnormalities are the most common electron microscopic changes in skeletal muscles. ${ }^{3}$

Diagnosis of TPP is based on clinical and biochemical evidence of hyperthyroidism and hypokalemia in a patient with a history of recurrent episodes of proximal muscle weakness, affecting mainly the lower limbs, without a family history of this disorder. The severity of attacks varies from mild weakness to quadriplegia or total paralysis. Bulbar, respiratory and ocular muscles are rarely affected. In the majority of patients, deep tendon reflexes are markedly diminished or absent. Cognitive and sensory functions remain normal. The onset of paralytic attacks usually coincides with the onset of hyperthyroidism, although symptoms, if present, are often mild. ${ }^{1,3}$ Some electrocardiogram features that can suggest a diagnosis of TPP are the triad of sinus tachycardia attributable to the hyperadrenergic state, prolonged QT-U interval attributable to hypokalemia, and a paradoxically prolonged PR interval due to the thyrotoxicosis. ${ }^{12}$

The differential diagnosis for TPP includes familial hypokalemic periodic paralysis (FHPP). Both disorders are identical in their clinical presentation, but TPP is rarely associated with a positive family history and has a later onset of presentation than FHPP. ${ }^{7,13}$ The recurrent attacks with normal plasma potassium levels between attacks distinguish 
periodic paralysis from other causes of hypokalemic paralysis. Moreover, mutations in the ionic channel genes such as CACNA1S, SCN4A and KCNE3 have been reported in the FHPP. 5

Definitive treatment of TPP consists in the management of thyrotoxicosis by medical therapy, surgery or radioactive iodine therapy. Treatment for an acute attack is potassium administration, but excessive doses of potassium can lead to hyperkalemia once potassium shifts to the extracellular space. ${ }^{6}$ No correlation between potassium dose administered and recovery time was observed. To prevent attacks until euthyroid state is achieved, a useful therapy is the administration of a $\beta$-adrenergic blocker like propranolol. Other preventive measures that may be effective include a low-carbohydrate diet and potassium-sparing diuretics. ${ }^{13}$ Use of potassium supplements is not useful for prophylaxis against further paralytic attacks, and it should not be given to patients between episodes. ${ }^{14}$

\section{Conclusion}

In summary, episodes of periodic paralysis usually precede the diagnosis of thyroid dysfunction and do not recur once euthyroidism is achieved. Therefore, it is necessary that an early diagnosis of TPP is made to administer definitive treatment and prevent morbidity and mortality, mainly due to fatal arrhythmias. The presence of acute paralysis, especially with hypokalemia, should prompt the clinician to consider TPP as a cause and evaluate thyroid function.

\section{References}

1. Kelley DE, Gharib H, Kennedy FP, Duda RJ Jr, McManis PG. Thyrotoxic periodic paralysis. Report of 10 cases and review of electromyographic findings. Arch Intern Med 1989;149:2597-2600.

2. Kodali VR, Jeffcote B, Clague RB. Thyrotoxic periodic paralysis: a case report and review of the literature. J Emerg Med 1999; 17:43-45.

3. Magsino CH Jr, Ryan AJ Jr. Thyrotoxic periodic paralysis. South Med J 2000;93:996-1003.

4. Laroia ST, Zaw KM, Ganti AK, Newman W, Akinwande AO. Amiodarone-induced thyrotoxicosis presenting as hypokalemic periodic paralysis. South Med J 2002;95: 1326-1328.

5. Tagami T, Usui T, Shimatsu A, Naruse M. Toxic thyroid adenoma presenting as hypokalemic periodic paralysis. Endocr J 2007; 54:797-803.

6. Manoukian M, Foote JA, Crapo LM. Clinical and metabolic features of thyrotoxic periodic paralysis in 24 episodes. Arch Intern Med 1999;159:601-606.

7. Seshadri P, Frank KD, Iqbal N. Thyrotoxic hypokalemic periodic paralysis in a Native American patient: case report and literature review. Endocr Pract 2002;8:362-363.

8. Wongraoprasert S, Buranasupkajorn P, Sridama V, Snabboon T. Thyrotoxic periodic paralysis induced by pulse methylprednisolone. Intern Med 2007;46:1431-1433.

9. Brown JD, Kangwanprasert M, Tice A, Melish J. Thyrotoxic periodic paralysis in a Polynesian male following highly active antiretroviral therapy for HIV infection. Hawaii Med J 2007;66:60, 62-63.
10. Cesur M, Gursoy A, Avcioglu U, Erdogan MF, Corapcioglu D, Kamel N. Thyrotoxic hypokalemic periodic paralysis as the first manifestation of interferon-alpha-induced Graves disease. J Clin Gastroenterol 2006;40:864-865.

11. Lichtstein DM, Arteaga RB. Rhabdomyolysis associated with hyperthyroidism. Am J Med Sci 2006;332:103-105.

12. Goldberger ZD. Images in cardiovascular medicine. An electrocardiogram triad in thyrotoxic hypokalemic periodic paralysis. Circulation 2007;115:e179-e180.

13. Ober KP. Thyrotoxic periodic paralysis in the United States. Report of 7 cases and review of the literature. Medicine (Baltimore) 1992;71:109-120.

14. Kung AW. Clinical review: thyrotoxic periodic paralysis: a diagnostic challenge. J Clin Endocrinol Metab 2006;91: 2490-2495.

\section{Author Affiliations}

M. J. Barahona

Department of Endocrinology and Nutrition

Hospital Sant Pau

Autonomous University of Barcelona (UAB)

Spain

\section{Vinagre}

Department of Endocrinology and Nutrition

Hospital Sant Pau

Autonomous University of Barcelona (UAB)

Spain

L. Sojo

Department of Endocrinology and Nutrition Hospital Sant Pau

Autonomous University of Barcelona (UAB)

Spain

J. M. Cubero

Department of Endocrinology and Nutrition

Hospital Sant Pau

Autonomous University of Barcelona (UAB)

Spain

Antonio Pérez

Department of Endocrinology and Nutrition Hospital Sant Pau

Autonomous University of Barcelona (UAB)

Spain 